\title{
Analysis of Coordinates Decoupling of Multi-Dimensional Precision Optical Adjusting Frame in Optical Alignment
}

\author{
Yong Luo ${ }^{1}$ \\ ${ }^{1}$ School of Intelligent Systems Science and Engineering, Jinan University, Zhuhai, China \\ Correspondence: Yong Luo, School of Intelligent Systems Science and Engineering, Jinan University, No.206 \\ Qianshan Road, Zhuhai, 519070, Guangdong Province, China.
}

Received: March 24, 2020

Accepted: April 20, 2020

Online Published: April 22, 2020

doi:10.5539/mas.v14n5p29

URL: https://doi.org/10.5539/mas.v14n5p29

\begin{abstract}
Information technology has much promoted the development of optical technology. With the help of computer technology, people can design and manufacture more complex optical systems than before, to obtain ideal imaging quality. The complexity of the optical system brings enormous challenges to optical alignment. Optical system alignment is the crucial link of transforming the excellent optical design into instruments with good performance in reality. Precision alignment of an optical system requires precise adjustment of each component's degree of freedom using a specific adjusting mechanism. Due to the quantification and compensation correction for the coordinates coupling relationship among each dimension adjusting freedom of the adjusting frame can not be carried out. Generally, the coordinates coupling problem is usually ignored in optical system alignment, to cause the optical adjustment error. This paper carries out an analysis for the coupling relationship among each dimension motion freedom of multi-dimensional precision optical adjusting frame in details by mathematical modeling and simulation, the decomposed transformation for each dimension adjusting mount of multi-dimensional precision optical adjusting frame, and the compensation correction for the coordinates coupling among each dimension adjusting mount. The test results show that this method can effectively reduce the difference between the actual mechanical adjusting mount and the expected optical adjusting mount, to achieve more accurate optical adjustment.
\end{abstract}

Keywords: optical alignment, precision optical adjusting frame, coordinate coupling, decoupling, compensation, and correction

\section{Introduction}

Optical instruments play an essential role in exploring both the micro and macro fields (Yang, 2005). With the development of modern optics, the requirements for optical systems are increasing, and the system structure is becoming more complex (Zhou, 2016). Optical systems with large linear fields of view, image quality close to the diffraction limit, large relative apertures and no central occlusion are required in some applications (Chen, 2013). Information technology has much promoted the development of optical technology. With the help of computer technology, people can design and manufacture more complex optical systems than ever before, to obtain ideal imaging quality (Yao, Hu \& Jiang, 2019). In space remote sensors, engineers have begun to use off-axis four-reflection optical systems with complex structures but excellent performance. The complexity of the optical system brings a great challenge to optical alignment (Chen \& Fu, 2003). The level of alignment accuracy directly affects the final imaging quality, and the optical alignment has become the critical link to determine whether the abstract design can be converted into equipment with excellent performance (Gu, 2016). For misaligned optical systems, the primary correction idea is to find out the misalignment state of each element of the optical system, that is, the deviation between the position of each component and the designed ideal position, including the direction of variation and the amount of variation. By adjusting the multi-degree of freedoms of the optical component to eliminate the coupling aberration. And thus, after each cycle of alignment, each element in the optical system is closer to the designed position state than before (Liu, Li \& Lv, 2005). The alignment process requires each component to have a corresponding adjustment mechanism for high accuracy adjustment.

In the optical alignment experiment, the adjustment of optical components is the adjustment for one or more freedom in six freedom (Figoski, Shrode \& Moore, 1989). The different basic design concepts of the optical 
adjusting frame are all for achieving the adjustment for one or more freedom and ensuring the accuracy of the alignment. An optical experiment, the five-dimensional adjusting frame for adjusting five freedom of the optical components is commonly used (Egdall, 1985).

For the five-dimensional adjusting frame, due to the restrictions of the system installation space, the requirements of the system alignment state, the mechanical structure design difficulty and the limits of processing processes, they both can not be orthogonal among the three-dimensional translation adjusting freedom of the adjusting frame; the rotary center of two-dimensional rotational freedom and the optical center of the optical components can not coincide (Kim, S, Yang, Lee \& Kim, S.W, 2007); therefore, when carrying out the adjustment for five freedom, the coordinates coupling is existed among each dimension adjusting mount, that is to say, one dimension is adjusted, another one dimension even several dimensions will be changed (Sun, $\mathrm{Lv}$, Yao \& Liu, 2014).

Generally, in the optical system alignment experiment, due to the quantification and compensation correction for the coordinates coupling relationship among the adjusting mount of each dimension can not be carried out, the coordinates coupling problem is usually ignored (Zhang et al., 2015). However, the ignored coordinates coupling relationship will cause the difference exists between the actual mechanical adjusting mount and the expected value of optical adjusting mount ( $\mathrm{Li}, \mathrm{Fu}$, Wang \& Duan, 2013); therefore, the convergence speed in the optical system alignment process is also affected, make the system alignment process becomes slower even diverges.

Combined with the optical alignment process of a specific off-axis three-reflection optical system, this paper analyzes the coupling relationship between each dimensional freedoms of a multi-dimensional precision optical frame through mathematical modeling and simulation, decomposes and transforms the adjusting mount of multi-dimensional optical adjusting frame, and compensates and corrects the coordinate coupling between the two. Also, high accuracy laser tracker and theodolite are used to evaluate the accuracy of the three-dimensional translation adjusting mount and the two-dimensional rotation regulating mount before and after the decoupling process, respectively. Test results show that the method proposed in this paper can effectively reduce the difference between the actual mechanical adjustment and the expected optical adjustment, thus achieving more accurate optical change.

\section{Establishing the Optical Alignment Environment}

A specific off-axis three-reflection optical system is designed to have the image quality approaching to the diffraction limit, the position accuracy of each optical component has a very high requirement: the location accuracy of displacement must be reached to micrometer level, the location accuracy of the angle must be entered to arcsecond level, to achieve good system alignment results, the computer-aided alignment environment of the off-axis three-reflection optical system is established as shown below:

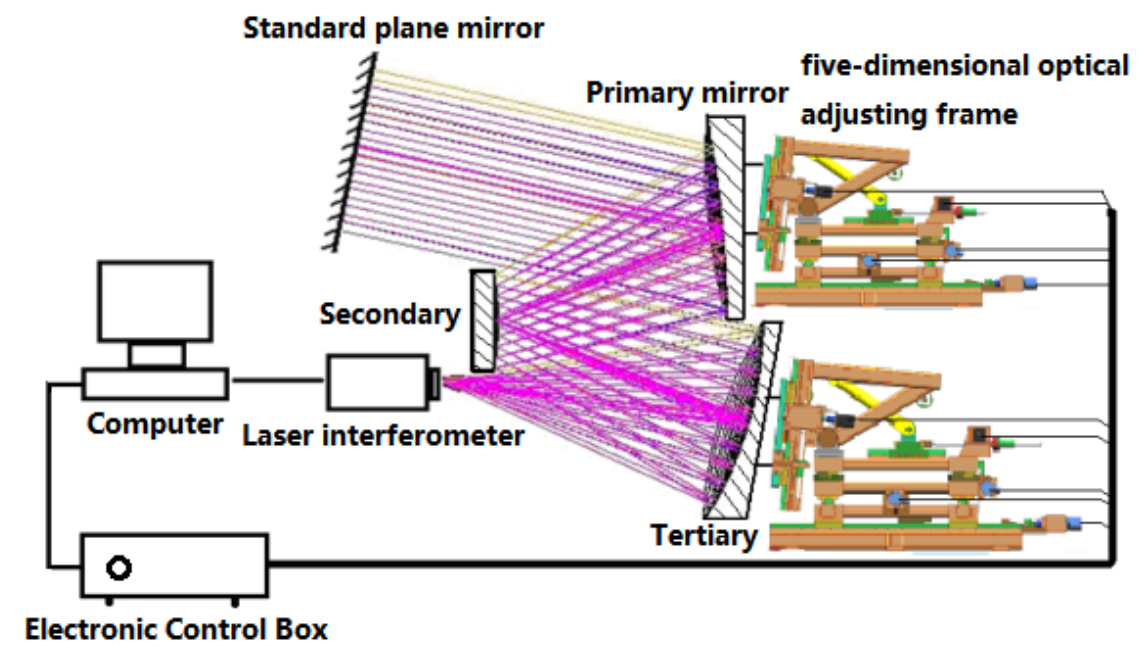

Figure 1. Diagram of computer-aided alignment of the three-reflection optical system 
As shown in Figure1, the imaging quality of the optical system is obtained by self-collimating interferometry, and the aberration of each field of view is obtained by ZYGO GPI laser interferometer; the primary mirror and the tertiary mirror of the system are precisely adjusted by two self-developed five-dimensional precision electric adjusting frames; the motion of the two five-dimensional precision electric adjusting frames are controlled by a computer through a multi-dimensional electronic control box.

\section{Mathematical Model}

The electrical precision five-dimensional optical adjusting frame is designed and developed for the precision alignment process of this system. The driving part of the adjusting frame uses a stepping motor with precision deceleration lead screw for high-precision movement. Due to the requirements of the system alignment state, as well as the restrictions of mechanical structure design and the processing process, a certain degree of coordinates coupling phenomenon exists among the five-dimensional adjusting freedom of this precision five-dimensional optical adjusting frame; to analyze the coordinates coupling relationship among each dimension, carry out the simplified model for this five-dimensional optical adjusting frame, as shown in Figure 2.

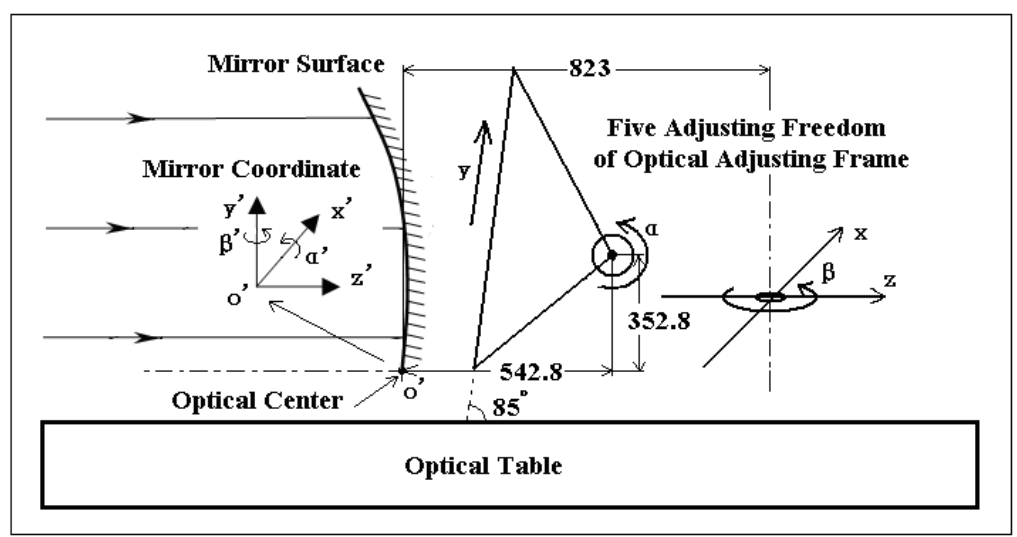

Figure 2. A simplified model for coordinate-coupling relation of the five-dimensional optical adjusting mount

In Figure 2, 1) In three-dimensional translation freedom of the adjusting frame, the $\mathrm{x}$-axis is perpendicular to z-axis; y-axis and z-axis present 85 degrees angle, and in YOZ plane that is perpendicular to the horizontal plane; 2) The rotary center of two-dimensional rotational freedom and the optical center of the optical components are not coincided, the horizontal distance between the rotating center of pitch adjusting mount a rotating around $\mathrm{x}$-axis and the optical center of the optical components has $542.8 \mathrm{~mm}$, the vertical distance is $352.8 \mathrm{~mm}$, the horizontal distance between the rotary center of the azimuth adjusting mount $\beta$ rotating around $y$-axis and the optical center of the optical components is $823 \mathrm{~mm}$; each dimension coordinates coupling relationship of this five-dimensional adjusting frame is shown in Table 1, therefore, in order to achieve high precision optical adjustment, need to carry out the coordinates decoupling processing for this five-dimensional precision adjusting frame.

Table 1. Coordinate-coupling relation of the five-dimensional optical adjusting mount

\begin{tabular}{ccc}
\hline Desired Adjustment & Single-Adjust & Actual Movement \\
\hline $\mathrm{X}$ & $\mathrm{X}$ & $\mathrm{X}$ \\
$\mathrm{Y}$ & $\mathrm{Y}$ & $\mathrm{Y}+\mathrm{Z}$ \\
$\mathrm{Z}$ & $\mathrm{Z}$ & $\mathrm{Z}$ \\
Pitch $\alpha$ & Pitch $\alpha$ & Pitch $\mathrm{a}+\mathrm{Y}+\mathrm{Z}$ \\
Azimuth $\beta$ & Azimuth $\beta$ & Azimuth $\beta+\mathrm{X}$ \\
\hline
\end{tabular}




\section{Coordinates Decoupling Analysis}

\subsection{Coordinates Decoupling Methods and Steps}

From Table 1, we know that the z-axis will be changed while adjusting the y-axis; y-axis and z-axis will be replaced when aligning the pitch mount $a ; x$-axis will be altered while adjusting the azimuth mount $\beta$. According to the coupling relationship among each dimension motion degree of freedom of this five-dimensional precision adjusting frame, it carries out the coordinates decoupling processing for this optical adjusting frame, designs the following methods and steps, as shown in Figure 3.

Step(1). By the decomposed transformation for the three-dimensional translation adjusting freedom of the five-dimensional optical adjusting frame, it is changed as the three-dimensional optical translation adjusting mount on the coordinate axis direction and under the rectangular coordinate system;

Step(2). The decomposition of the two-dimensional rotary adjusting mount is taken as the decomposed processing of the translation mount on the parallel axis direction and under the rectangular coordinate system and the rotational mount rotating around the parallel axis;

Step(3). Sum up the action (1) and (2), and solve the equations, set up the mapping relationship among the expected each dimension adjusting amount of the optical system and each dimension adjusting freedom of the adjusting frame under the rectangular coordinate system.

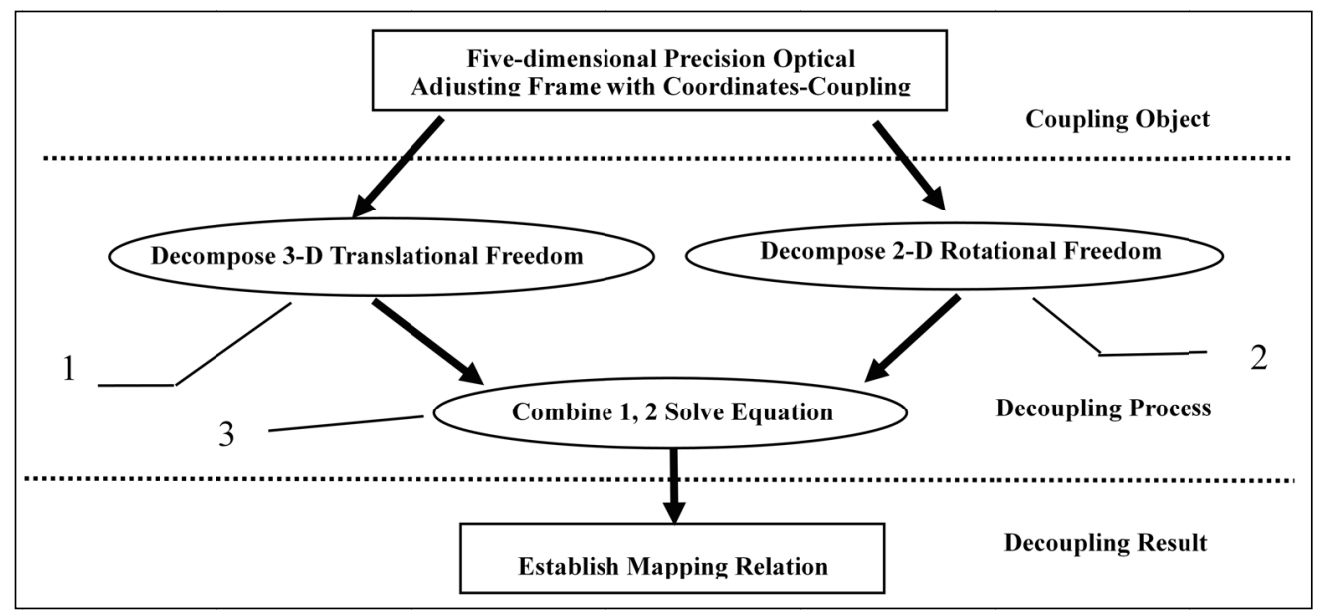

Figure 3. Procedure for coordinate-decoupling

\subsection{Coordinates Decoupling Analysis}

In the following, combine the step (1), (2) and (3), carry on the coordinates decoupling analysis for this five-dimensional optical adjusting frame:

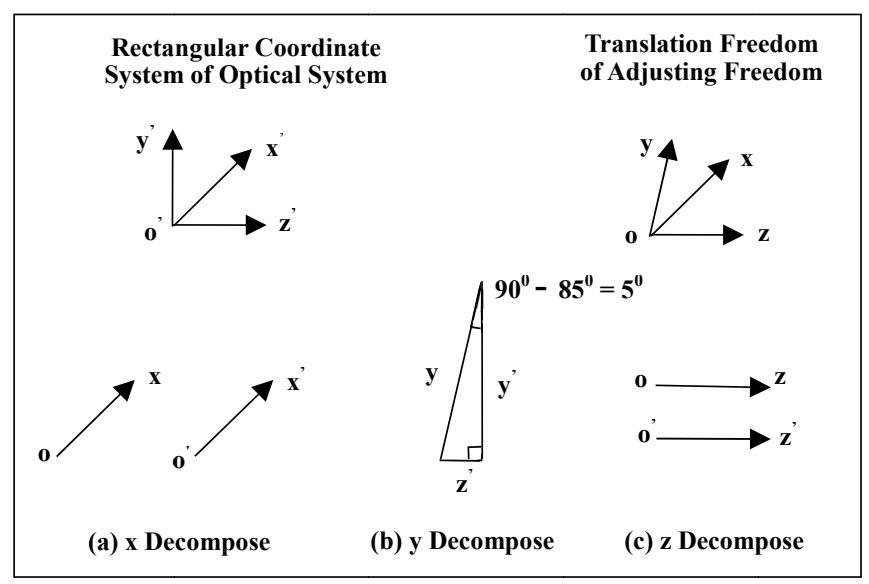

Figure 4. Schematic diagram of the decomposed transformation of the translation motion 
Firstly, carry on the decomposed change for the three-dimensional translation mount. As shown in Figure 4, decompose the three-dimensional translation adjusting freedom $\mathrm{x}, \mathrm{y}, \mathrm{z}$ to the three-dimensional optical rendering changing mount $x^{\prime}, y^{\prime}$ and $z^{\prime}$ on the coordinates axis direction and under the rectangular coordinate system:

$$
\left\{\begin{array}{l}
x^{\prime}=x \\
y^{\prime}=y \times \sin \left(85^{\circ}\right) \\
z^{\prime}=z+y \times \cos \left(85^{\circ}\right)
\end{array}\right.
$$

Later on, carry on the decomposed transformation for the two-dimension rotational mount. As shown in Figure 4, the little two-dimensional rotational adjusting mount in optical adjustment is decomposed to the translation mount on the coordinates axis direction, and the rotational mount around the coordinates axis under the rectangular coordinate system. The pitch mount a rotating around $\mathrm{x}$-axis is decomposed to the translation mount $y^{\prime}$ on $y^{\prime}$ axis direction, the translation mount $z^{\prime}$ on $z^{\prime}$ axis direction, and the rotational mount $a^{\prime}$ around $x^{\prime}$ axis under the rectangular coordinate system, that are respectively approximately shown as:

$$
\left\{\begin{array}{l}
\alpha^{\prime}=\alpha \\
r=\sqrt{352.8^{2}+542.8^{2}} \times \tan \left(\alpha^{\prime}\right) \approx \sqrt{352.8^{2}+542.8^{2}} \times \alpha \\
\theta=\arctan \left(\frac{352.8}{542.8}\right) \\
y^{\prime}=r \times \cos (\theta) \approx \sqrt{352.8^{2}+542.8^{2}} \times \alpha \times \cos \left[\arctan \left(\frac{352.8}{542.8}\right)\right] \\
z^{\prime}=r \times \sin (\theta) \approx \sqrt{352.8^{2}+542.8^{2}} \times \alpha \times \sin \left[\arctan \left(\frac{352.8}{542.8}\right)\right]
\end{array}\right.
$$

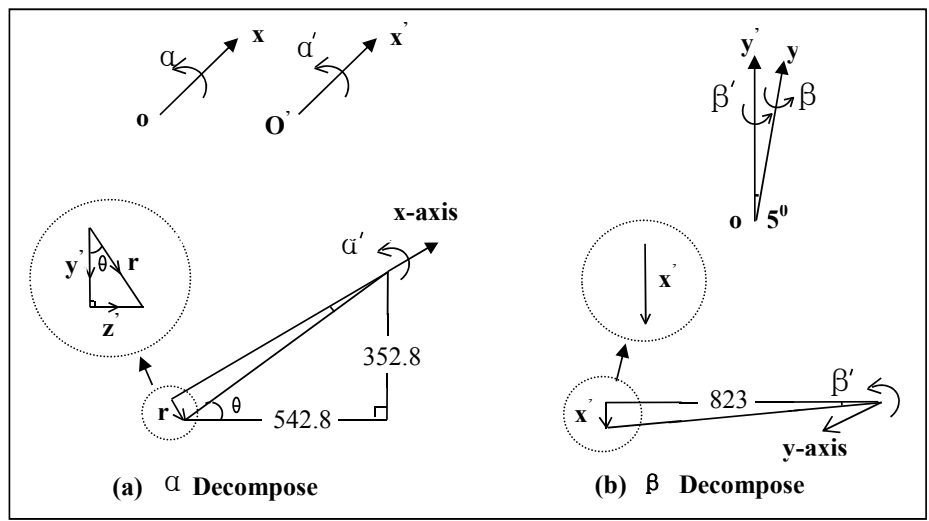

Figure 5. Schematic diagram of the decomposed transformation of the rotational motion

As shown in Figure 5, the little azimuth adjusting mount $\beta$ rotating around $y$-axis is decomposed to the rotational mount $\beta^{\prime}$ around $y^{\prime}$ axis under the rectangular coordinate system and the translation mount $x^{\prime}$ on $x^{\prime}$ axis direction that are respectively approximately shown as:

$$
\begin{aligned}
& \beta^{\prime} \approx \beta \times \cos \left(90^{\circ}-85^{\circ}\right)=\beta \times \cos \left(5^{\circ}\right) \\
& x^{\prime}=823 \times \tan \left(\beta^{\prime}\right) \approx 823 \times \beta \times \cos \left(5^{\circ}\right)
\end{aligned}
$$

Combine the above equations, solve the mapping relationship between the five-dimensional adjusting amount of the optical system and the five-dimensional adjusting freedom of the adjusting frame under the rectangular coordinate system, the solution result is shown as below: 


$$
\left\{\begin{array}{l}
x=x^{\prime}-823 \times \frac{\beta^{\prime}}{\cos \left(5^{0}\right)} \\
y=y^{\prime}+\sqrt{352.8^{2}+542.8^{2}} \times \alpha \times \cos \left[\arctan \left(\frac{352.8}{542.8}\right)\right] \\
z=z^{\prime}-y \times \cos \left(85^{0}\right)-\sqrt{352.8^{2}+542.8^{2}} \times \alpha \times \sin \left[\arctan \left(\frac{352.8}{542.8}\right)\right] \\
\alpha=\alpha^{\prime} \\
\beta=\frac{\beta^{\prime}}{\cos \left(5^{0}\right)}
\end{array}\right.
$$

According to the mapping relationship, transform the five-dimensional optical adjusting mount $x^{\prime}, y^{\prime}, z^{\prime}, a^{\prime}, \beta^{\prime}$ of the optical system to be achieved under the rectangular coordinate system to the adjusting mount of the five-dimensional adjusting degree of freedom $x, y, z, a, \beta$ of the adjusting frame, therefore, the coordinates decoupling of the five-dimensional adjusting mount of the five-dimensional optical adjusting frame is achieved, to enhance the accuracy of the optical adjustment.

Using the above ideas, coordinates decoupling processing is performed on the inter-coupling relationship between each degree of freedom of the optical adjustment frame. When the relative positional relationship of each dimension of the optical adjustment frame is unchanged, no matter the posture of the adjustment frame is changed or moved, the decoupling relationship will not change. The results of equation (5) are applicable.

\section{Actual Optical Alignment and Adjustment Accuracy Test}

In the actual optical alignment experiment, $\mathrm{PC}$ is used as the main control computer in the control of a five-dimensional electric precision optical alignment frame, and a five-dimensional adjustable stepper motor driver controller is specially developed. The two controllers communicate with each other through the RS232 interface. Five-dimensional stepper motor control box is composed of the communication module, main control microcontroller, wireless remote control module, stepper motor drive module, and power supply. To facilitate optical alignment, developed the computer-aided alignment integrated control software of the optical system using Visual Basic 6.0. The software functions include the misalignment calculation module and the motion control module of the adjusting frame. The motion control module of the adjusting frame can realize the step-by-step driving function and coordinate the decoupling driving function of the five-dimensional optical adjusting frame.

To verify the actual effect of the coordinates decoupling method as put forward by this paper, go on adopting the motion accuracy measurement for the three-dimensional translation adjusting freedom by combing Leica laser tracker with the accuracy of translation measurement of 50um and T-Probe, and the motion accuracy measurement for the two-dimensional rotation regulating immunity by Leica TM5100A theodolite with the precision of angular displacement measurement around \pm 0.5 arc second, as well as the adjusting accuracy comparison measurement for the effects before and after the coordinates decoupling of this five-dimensional optical adjusting frame, and evaluate the coordinates decoupling result among each dimension adjusting mount.

\subsection{Small Displacement Drive Test}

Firstly, in the stepping drive mode of each dimensional adjustment of the adjustment frame, the five-dimensional motor is directly driven by the motion command, three-dimension translation adjusting freedom are respectively regulated as $0.1 \mathrm{~mm}$, two-dimension rotary adjusting freedom are respectively regulated as $0.1^{\circ}$; secondly, in the parallel decoupling drive mode of each dimensional adjustment of the adjustment frame, the decomposed transformation is carried out for the decoupling result Equation (5), and then five dimension motors are respectively driven, and each dimension is regulated; the actual adjusting mount of the five aspects adjusting freedom under the optical center coordinate system of the reflector are respectively monitored by Leica laser tracker and Leica TM5100A theodolite, the results are shown in Table 2:

Table 2. Coordinate-decoupling result of small-displacement drive test

\begin{tabular}{ccccc}
\hline Each adjusting freedom & Movement (Before) & Errors & Movement (After) & Errors \\
\hline X:0.1 mm & $-1.29 \mathrm{~mm}$ & $-1390 \%$ & $0.09 \mathrm{~mm}$ & $-10.0 \%$ \\
Y:0.1 mm & $1.02 \mathrm{~mm}$ & $+920 \%$ & $0.08 \mathrm{~mm}$ & $-20.0 \%$ \\
Z:0.1 mm & $-0.49 \mathrm{~mm}$ & $-590 \%$ & $0.09 \mathrm{~mm}$ & $-10.0 \%$ \\
Pitch a: $0.1^{\circ}$ & $0.099^{\circ}$ & $-1.0 \%$ & $0.098^{\circ}$ & $-2.0 \%$ \\
Azimuth $\beta: 0.1^{\circ}$ & $0.098^{\circ}$ & $-2.0 \%$ & $0.094^{\circ}$ & $-6.0 \%$ \\
\hline
\end{tabular}


From Table2, When driving with small displacement, the conclusions are obtained as follows: before carrying out the coordinates decoupling correction, the adjusting accuracy of the pitch mount a and the Azimuth mount $\beta$ of the two-dimensional rotational freedom of the five-dimensional adjusting frame are right, after the coordinate decoupling correction, the accuracy of the rotation adjustment degree of freedom has deteriorated to a certain extent. Three dimensions translation adjusting error are respectively arrived at: $-1390 \%,+920 \%$, and $-590 \%$, and the accuracy is inferior and completely unusable; after the coordinates decoupling correction, three dimensions translation adjusting error are respectively reduced to $-10.0 \%,-20.0 \%$ and $-10.0 \%$, the adjusting accuracy is enhanced obviously.

After the coordinate decoupling correction, the accuracy of the rotation adjustment degree of freedom has deteriorated to a certain extent; the efficiency of the three dimensions translation adjustment degree of freedom has been enhanced obviously. But, there are still glaring residual errors, and the number of movements is all insufficient. The reason may be caused by the existence of a small amount of axial clearance in the precision deceleration lead screw. However, in general, the precision of the little displacement drive of the adjusting frame has reached the accuracy requirement of the adjustment after coordinate decoupling correction.

\subsection{Massive Displacement Drive Test}

Firstly, in the stepping drive mode of each dimensional adjustment of the adjustment frame, the five-dimensional motor is directly driven by the motion command, three-dimension translation adjusting freedom are respectively regulated as $1.0 \mathrm{~mm}$, two-dimension rotary adjusting freedom are respectively monitored as $1.0^{\circ}$; secondly, in the parallel decoupling drive mode of each dimensional adjustment of the adjustment frame, the decomposed transformation is carried out for the decoupling result Equation (5), and then five dimension motors are respectively driven, and each dimension is regulated; the actual adjusting mount of the five aspects adjusting freedom under the optical center coordinate system of the reflector are respectively monitored by Leica laser tracker and Leica TM5100A theodolite, the results are shown in Table 3:

Table 3. Coordinate-decoupling result of massive displacement drive test

\begin{tabular}{ccccc}
\hline Each adjusting freedom & Movement (Before) & Errors & Movement (After) & Errors \\
\hline X: $1.0 \mathrm{~mm}$ & $-13.30 \mathrm{~mm}$ & $-1430 \%$ & $1.06 \mathrm{~mm}$ & $+6.0 \%$ \\
Y: $1.0 \mathrm{~mm}$ & $10.48 \mathrm{~mm}$ & $+948 \%$ & $1.03 \mathrm{~mm}$ & $+3.0 \%$ \\
Z:1.0 mm & $-5.15 \mathrm{~mm}$ & $-615 \%$ & $0.97 \mathrm{~mm}$ & $-3.0 \%$ \\
Pitch a: $1.0^{\circ}$ & $1.021^{\circ}$ & $-2.1 \%$ & $1.014^{\circ}$ & $+1.4 \%$ \\
Azimuth $\beta: 1.0^{\circ}$ & $0.992^{\circ}$ & $-0.8 \%$ & $0.997^{\circ}$ & $-0.3 \%$ \\
\hline
\end{tabular}

From Table3, When driving with massive displacement, the conclusions are obtained as follows: before carrying out the coordinates decoupling correction, except the adjusting accuracy of the pitch, mount $a$ and the azimuth mount $\beta$ of the two-dimensional rotational freedom of the five-dimensional adjusting frame are right, after the coordinate decoupling correction, the accuracy of the rotation adjustment degree of freedom has been improved to a certain extent. Three dimensions translation adjusting error are respectively arrived at: $-1430 \%,+948 \%$, and $-615 \%$, the accuracy is inferior and completely unusable; after the coordinates decoupling correction, three dimensions translation adjusting error are respectively reduced to $+6 \%,+3 \%$, and $-3 \%$, the adjusting accuracy is enhanced obviously and meet the requirements of the alignment accuracy.

\section{Conclusion}

Combined with the optical alignment process of a specific off-axis three-reflection optical system, this paper respectively carries out the decomposed transformation for the three-dimensional translation adjusting mount, and the two-dimensional rotary adjusting mount of the five-dimensional optical adjusting frame, and make the decoupling analysis for the small adjusting mount of each dimension in optical adjustment by the short linear approximation method. The actual test shows that this method can reduce the coordinates coupling phenomenon among each aspect adjusting mount of the five-dimensional optical adjusting frame effectively, can prompt the actual optical adjusting mount is more coincided with the expected adjusting mount, to achieve the more accurate adjustment of each dimension adjusting mount in the optical precision alignment process, make the system alignment process becomes faster.

Also, the decoupling idea proposed in this paper can be used for coordinate transformation and decoupling 
operation of multi-dimensional optical adjustment frame in other similar situations to reduce the difference between the actual mechanical adjustment mount and the desired optical adjustment mount, thereby achieving more accurate optical adjustment.

\section{Acknowledgments}

This work is a result of research supported by "the Fundamental Research Funds for the Central Universities" NO.21617360, as well as "the Research and Innovation Funds Project of Jinan University" NO.21617360. Besides, we thank XIAO Jing for her linguistic assistance during the preparation of this manuscript.

\section{References}

Chen, J. J. (2013). Application of computer aided alignment technique to a three-mirror off-axis optical system. University of Chinese Academy of Sciences, Beijing, China.

Chen, X. L., Fu, D. Y. (2003). Solutions for space optical remote sensor with large aperture and ultra high resolution. Spacecraft Recovery \& Remote Sensing, 24(4), 19-24.

Egdall, I. M. (1985). Manufacture of a three-mirror wide-field optical system. Optical Engineering, 24(2), 285-289. https://doi.org/10.1117/12.7973470.

Figoski, J. W., Shrode, T. E., Moore, G. F. (1989). Computer-aided alignment of a wide-field, three-mirror, unobscured, high-resolution sensor. Proceedings of SPIE- The International Society for Optical Engineering, 1049, 166-177. https://doi.org/10.1117/12.951421.

Gu, Z. Y. (2016). Misalignment corrections in reflective telescopes. University of Chinese Academy of Sciences, Beijing, China.

Kim, S., Yang, H. S., Lee, Y. W., Kim, S. W. (2007). Merit function regression method for efficient alignment control of two-mirror optical systems. Optics Express, 15(8), 5059-5068. https://doi.org/10.1364/OE.15. 005059.

Li, X. Y., Fu, X., Wang, P., Duan, X. T. (2013). Alignment method of aspheric optical reflector. Journal of Applied Optics, 34(3), 498-502. http://doi.org/10.5768/JAO201334.0305001.

Liu, L., Li, J. L., Lv, Q. T. (2005). Research on adjusting device of large aperture reflective optics system. Optics and Precision Engineering, 13(z1), 134-137.

Sun, J. W., Lv, T. Y., Yao, L. S., Liu, J. (2014). Design and assembly of transmitter-telescope. Optics and Precision Engineering, 22(2), 369-375. https://doi.org/10.3788/OPE.20142202.0369

Yang, X. F. (2005). Study on the computer-aided alignment of three-mirror optical system. Changchun Institute of Optics, Fine Mechanics and Physics, Chinese Academy of Sciences, Changchun, China.

Yao, M. Y., Hu, Z. W., Jiang, H. J. (2019). Alignment Metrology of an Off-axis Large-diameter Reflective Collimator. ACTA ASTRONOMICA SINICA, 60(2), 15-1 - 15-9. http://doi.org/10.15940/j.cnki.0001-5245. 2019.02.015.

Zhang, X. M., Jiang, F., Kong, L. Y., Li, Y. X., Liu, Y. C., Zhang, J. L., ... Zhong, L. P. (2015). Research on optical alignment technology for Cassegrain system. Journal of Applied Optics, 36(4), 526-530. https://doi.org/10.5768/JAO201536.0401006

Zhou, L. F. (2016). Study on the alignment technique of large aperture reflecting telescope on-line. University of Chinese Academy of Sciences (Institute of Optics and Electronics), Chengdu, China.

\section{Copyrights}

Copyright for this article is retained by the author(s), with first publication rights granted to the journal.

This is an open-access article distributed under the terms and conditions of the Creative Commons Attribution license (http://creativecommons.org/licenses/by/3.0/). 